\title{
Some Uniqueness Theorems for First Order Hyperbolic Systems
}

\author{
By \\ Hitoshi IshII*, Yoshinori SAgISAKA* \\ and Masayoshi Tsutsumi*
}

\section{Introduction}

It has been shown that if a solution $u$ of the Klein-Gordon equation $\left(\frac{\partial^{2}}{\partial t^{2}}-\Delta+\alpha\right) u(x, t)=0, x \in \mathbb{R}^{n}, \alpha>0$, vanishes on a half characteristic cone and its energy integral is finite, then $u$ vanishes identically. This uniqueness theorem was originally proved by Segal [8] in case $n=1$ and extended to the general case $(n>1)$ by Goodman [1] and Morawetz [7].

Taniguchi has shown in [9] that the same uniqueness theorem is valid for some first order symmetric hyperbolic systems in the case when $n=1$.

In this paper, we intend to extend his result to the case when $n>1$ and to more general hyperbolic systems, which, in particular, enables us to discuss the above uniqueness of solutions of the Dirac equation.

The authors wish to express their sincere thanks to Prof. R. Iino for his encouragement and kind suggestions.

After this paper was submitted, the authors were informed of the Hörmander's work entitled "Lower bounds at infinity for solutions of differential equations with constant coefficients" (Israel J. Math., 16, 103-116 (1973)) by Prof. M. Murata. His results contain a part of Theorem 2.1 in this paper. The authors thank to Prof. M. Murata for his kind announcement.

Communicated by S. Matsuura, October 14, 1974.

* Department of Applied Physics, Waseda University, Tokyo. 


\section{Main Theorem}

We shall consider the first order hyperbolic system

$$
\frac{\partial u}{\partial t}=\sum_{j=1}^{n} A_{j} \frac{\partial u}{\partial x_{j}}+L u, \quad x=\left(x_{1}, \ldots, x_{n}\right) \in \mathbb{R}^{n}, t \in \mathbb{R}^{1}
$$

where $u$ is a $\mathbb{C}^{N}$-valued function in $\mathbb{R}^{n+1}$, and $A_{j}$ and $L$ are $N \times N$ constant matrices.

Throughout this paper, we make the following assumptions:

(i) All the eigenvalues of the matrix $\sum_{j=1}^{n} A_{j} \xi_{j}+i L$ are real for all $\xi$ $=\left(\xi_{1}, \ldots, \xi_{n}\right) \in \mathbb{R}^{n}$. These eigenvalues will be designated by $\lambda_{j}(\xi)\left(\lambda_{1}(\xi)\right.$ $\left.\leqq \lambda_{2}(\xi) \leqq \cdots \leqq \lambda_{N}(\xi)\right)$.

(ii) The matrix $\sum_{j=1}^{n} A_{j} \xi_{j}+i L$ is uniformly diagonalizable, i.e., there exists a nonsingular matrix $P(\xi)$ such that

$$
\|P(\xi)\|,\left\|P(\xi)^{-1}\right\| \leqq C \quad \text { for all } \xi \in \mathbb{R}^{n}
$$

and

$$
P(\xi)\left(\sum_{j=1}^{n} A_{j} \xi_{j}+i L\right) P(\xi)^{-1}=\left(\begin{array}{ccc}
\lambda_{1}(\xi) & & 0 \\
& \ddots & \\
0 & & \lambda_{N}(\xi)
\end{array}\right) \equiv \Lambda(\xi),
$$

where $\|\cdot\|$ denotes the matrix norm.

By $L^{2}$-solution we shall mean a solution $u(x, t)$ of the system (2.1) in the sense of distributions in $\mathbb{R}^{n+1}$ which is a continuous function in the variable $t$ with values in $L^{2}\left(\mathbb{R}^{n}\right)$ (i.e., $u \in C\left(\mathbb{R}^{1} ; L^{2}\left(\mathbb{R}^{n}\right)\right)$ ). Under the assumption (i) and (ii), for any $u_{0} \in L^{2}\left(\mathbb{R}^{n}\right)$, the unique $L^{2}$-solution $u$ satisfying $u(x, 0)=u_{0}(x)$ exists and is represented as

$$
\begin{aligned}
\hat{u}(\xi, t) & =\exp \left(-i t\left(\sum_{j=1}^{n} A_{j} \xi_{j}+i L\right)\right) \hat{u}_{0}(\xi) \\
& =P(\xi)^{-1} e^{-i t \Lambda(\xi)} P(\xi) \hat{u}_{0}(\xi), \quad t \in \mathbb{R}^{1},
\end{aligned}
$$

via the Fourier transformation where ^ denotes the Fourier transform with respect to $x$ which is defined by

$$
\hat{f}(\xi)=(2 \pi)^{-n} \int_{\mathbb{R}^{n}} e^{i x \cdot \xi} f(x) d x \quad \text { for } \quad f \in C_{0}^{\infty}\left(\mathbb{R}^{n}\right)
$$


and, in general, by the extension of this mapping $f \rightarrow \hat{f}$ by the continuity in $L^{2}\left(\mathbb{R}^{n}\right)$.

By the representation (2.2), using the Parseval's relation, we obtain

$$
\|u(\cdot, t)\|_{L^{2}\left(\mathbf{R}^{n}\right)} \leqq C\left\|u_{0}\right\|_{L^{2}\left(\mathbf{R}^{n}\right)}
$$

and

$$
\left\|u_{0}\right\|_{L^{2}\left(\mathbf{R}^{n}\right)} \leqq C\|u(\cdot, t)\|_{L^{2}\left(\mathbf{R}^{n}\right)}
$$

for $t \in \mathbf{R}^{1}$

Thus we know that the one-parameter family of operators $G(t)$, $t \in \mathbf{R}^{1}$, defined by $G(t) u_{0}=u(\cdot, t)$ forms a equi-bounded group of class $\left(C_{0}\right)$ on $L^{2}\left(\mathbf{R}^{n}\right)$ (Yosida [10]).

It is known that the singular support of the function $\lambda_{j}$ is a set of measure zero in $\mathbf{R}^{n}$ for each $j=1, \ldots, N$.

It follows from the assumption (i) that all the eigenvalues of the matrix $\sum_{j=1}^{n} A_{j} \xi_{j}$ are also real for $\xi \in \mathbb{R}^{n}$. We denote them by $\sigma_{j}(\xi), j$ $=1, \ldots, N$, and put

$$
\sigma=\max _{\substack{|\xi|=1 \\ 1 \leqq j \leqq N}} \sigma_{j}(\xi)
$$

We assume below that $\sigma>0$.

For a measurable set $M$, meas $M$ will denote the Lebesgue measure of $M$. We denote the gradient of $f$ by $\nabla f$.

We now state our

Theorem 2.1. Let $D=\underset{1 \leqq j \leqq N}{\bigcup}$ sing supp $\lambda_{j}$ and $E=\underset{1 \leqq j \leqq N}{\bigcup}\left\{\xi \in \mathbb{R}^{n} \backslash D\right.$; $\left.\left|\nabla \lambda_{j}(\xi)\right|=\sigma\right\}$. Suppose that

$$
\text { meas } E=0 \text {. }
$$

If a $L^{2}$-solution $u$ of the system (2.1) vanishes almost everywhere in the cone $K=\left\{(x, t) \in \mathbb{R}^{n} \times(0, \infty) ;|x|<\sigma t\right\}$, then $u$ vanishes a.e. in $\mathbb{R}^{n+1}$.

Remarks. In the above statement, we may replace the cone $K$ by the cones $K_{+}=\left\{(x, t) \in \mathbf{R}^{n+1} ; t>t_{0},\left|x-x_{0}\right|<\sigma\left(t-t_{0}\right)\right\} \quad$ or $\quad K_{-}=\{(x, t)$ $\left.\in \mathbf{R}^{n+1} ; t<t_{0},\left|x-x_{0}\right|<\sigma\left(t_{0}-t\right)\right\}$ with the vertex at $\left(x_{0}, t_{0}\right)$. When $n=1$, 
if meas $E>0$, then the conclusion of Theorem 2.1 does not hold any longer. This fact will be shown in section 5 .

We shall prove Theorem 2.1 in the next section.

Let $H_{\text {loc }}^{s}\left(\mathbb{R}^{n+1}\right), s \geqq 0$, be the space of all functions $f$ such that $f \in H^{s}(\Omega)$ (see Lions-Magenes [4]) for every open ball $\Omega \subset \mathbb{R}^{n+1}$. Denote by $d S$ the element of area on the surface $\partial K=\left\{(x, t) \in \mathbb{R}^{n} \times(0, \infty)\right.$; $|x|=\sigma t\}$. Let $L_{\text {loc }}^{2}(\partial K)$ be the space of all functions $f$ defined on $\partial K$ which are square integrable with respect to $d S$ in any open relatively compact subset of $\partial K$. Then both $H_{\text {loc }}^{s}\left(\mathbb{R}^{n+1}\right)$ and $L_{\text {loc }}^{2}(\partial K)$ are Fréchet spaces with obvious topologies.

The trace theorem asserts that for $s>\frac{1}{2}$, the trace operator $\gamma$ is continuous from $H_{\text {loc }}^{s}\left(\mathbb{R}^{n+1}\right)$ to $L_{\text {loc }}^{2}(\partial K)$. As for the trace operator and the trace theorem, we refer to Lions-Magenes [4].

Corollary 2.2. Under the hypothesis (iii), if a $L^{2}$-solution $u$ belongs to $H_{\mathrm{loc}}^{s}\left(\mathbf{R}^{n+1}\right)$ for some $s>\frac{1}{2}$ and if $\gamma u$ vanishes a.e. on $\partial K$, then $u=0$ a.e. in $\mathbb{R}^{n+1}$.

Proof. In view of Theorem 2.1, it suffices to prove that $u$ vanishes a.e. in $K$.

Choose a function $\psi \in C_{0}^{\infty}\left(\mathbb{R}^{n+1}\right)$ satisfying $\int_{\mathbb{R}^{n+1}} \psi(x, t) d x d t=1$ and set $\psi_{\delta}(x, t)=\delta^{-n-1} \psi(x / \delta, t / \delta)$ for $\delta>0$. Then $u_{\delta}=\psi_{\delta} * u$ (the convolution with respect to the variables $(x, t))$ is a smooth $L^{2}$-solution of the system (2.1) and tends to $u$ in $H_{\mathrm{loc}}^{s}\left(\mathbf{R}^{n+1}\right)$ as $\delta$ tends to zero. Henceforth, $B_{r}$ denotes the open ball in $\mathbb{R}^{n}$ centered at the origin whose radius equals to $r$.

For a given $T>0$ and any $\varphi \in C_{0}^{\infty}\left(B_{\sigma T}\right)$, let $v$ be the solution of the adjoint system of (2.1):

$$
\frac{\partial v}{\partial t}=\sum_{j=1}^{n} A_{j}^{*} \frac{\partial v}{\partial x_{j}}-L^{*} v, \quad x \in \mathbb{R}^{n}, t \in \mathbb{R}^{1}
$$

such that $v(x, T)=\varphi(x)$ if $x \in B_{\sigma T}$ and $v(x, T)=0$ otherwise, where $A_{j}^{*}$ and $L^{*}$ are the adjoint matrices of $A_{j}$ and $L$. Notice that the system (2.5) satisfies the conditions (i) and (ii) and that $v \in C^{\infty}\left(\mathbb{R}^{n+1}\right)$.

Since $u_{\delta}$ is a solution of (2.1), by (2.5), we have 


$$
\frac{\partial}{\partial t}\left\langle u_{\delta}, v\right\rangle-\sum_{j=1}^{n} \frac{\partial}{\partial x_{j}}\left\langle A_{j} u_{\delta}, v\right\rangle=0
$$

Let $T>\varepsilon>0, V=\left\{(x, t) \in \mathbb{R}^{n+1} ;|x| \leqq \sigma t, \varepsilon \leqq t \leqq T\right\} \quad$ and $\quad S=\left\{(x, t) \in \mathbb{R}^{n+1}\right.$; $|x|=\sigma t, \varepsilon \leqq t \leqq T\}$.

Integrating both sides of (2.6) over $V$ and using the Green's formula, we get

$$
\begin{aligned}
& \int_{\boldsymbol{B}_{\sigma \boldsymbol{T}}}\left\langle u_{\delta}(x, T), \varphi(x)\right\rangle d x-\int_{\boldsymbol{B}_{\sigma \varepsilon}}\left\langle u_{\delta}(x, \varepsilon), v(x, \varepsilon)\right\rangle d x \\
& -\int_{S} \frac{1}{\sqrt{1+\sigma^{2}}}\left(\sigma\left\langle u_{\delta}, v\right\rangle+\sum_{j=1}^{n} \frac{x_{j}}{|x|}\left\langle A_{j} u_{\delta}, v\right\rangle\right) d S=0 .
\end{aligned}
$$

Since $\gamma u=0$ a.e. in $S$, letting $\delta \rightarrow 0$, we see

$$
\int_{B_{\sigma T}}\langle u(x, T), \varphi(x)\rangle d x=\int_{B_{\sigma \varepsilon}}\langle u(x, \varepsilon), v(x, \varepsilon)\rangle d x .
$$

Since $u \in C\left(\mathbf{R}^{1} ; L^{2}\left(\mathbf{R}^{n}\right)\right)$ and $v \in C^{\infty}\left(\mathbf{R}^{n+1}\right)$,

$$
\int_{\boldsymbol{B}_{\sigma \boldsymbol{T}}}\langle u(x, T), \varphi(x)\rangle d x=\lim _{\varepsilon \rightarrow 0} \int_{\boldsymbol{B}_{\sigma \varepsilon}}\langle u(x, \varepsilon), v(x, \varepsilon)\rangle d x=0 .
$$

This implies that $u=0$ a.e. in $K$, which finishes the proof of the corollary.

\section{Proof of Theorem 2.1}

To prove the theorem, we shall need the following

Lemma 3.1. Let $D$ be a closed set of measure zero in $\mathbb{R}^{n}$ and $\lambda$ be a real-valued function defined in $\mathbb{R}^{n}$ which belongs to $C^{[n / 2]+2}\left(\mathbf{R}^{n} \backslash D\right)$. For $f \in L^{1}\left(\mathbf{R}^{n}\right) \cap L^{2}\left(\mathbf{R}^{n}\right)$, define

$$
g(x, t)=\int_{\mathbf{R}^{n}} e^{-i x \cdot \xi-i \lambda(\xi) t} f(\xi) d \xi, \quad t>0 .
$$

Let $A$ be a compact set in $\mathbf{R}^{n}, B$ be a measurable bounded set in $\mathbf{R}^{n}$ and set $F_{t}=\{a t+b ; a \in A, b \in B\}, t>0$. If meas $\left\{\xi \in \mathbb{R}^{n} \backslash D ;-\nabla \lambda(\xi) \in A\right\}$ $=0$, then 


$$
\lim _{t \rightarrow \infty}\|g(\cdot, t)\|_{L^{2}\left(F_{t}\right)}=0
$$

The proof of this lemma will be given after the proof of the theorem.

Proof of Theorem. Let $u$ be a $L^{2}$-solution of the system (2.1) which vanishes a.e. in $K$. Put $u_{0}(x)=u(x, 0)$. For a given $\varepsilon>0$, we choose a function $v_{0}$ in $C_{0}^{\infty}\left(\mathbf{R}^{n}\right)$ satisfying

$$
\left\|u_{0}-v_{0}\right\|_{L^{2}\left(\mathbf{R}^{n}\right)}<\varepsilon
$$

We now choose $R$ so large that the support of $v_{0}$ is contained in the ball $B_{R}$. Denoting by $v$ the $L^{2}$-solution of $(2.1)$ such that $v(x, 0)=v_{0}(x)$, by (2.3), we have

$$
\|u(\cdot, t)-v(\cdot, t)\|_{L^{2}\left(\mathbf{R}^{n}\right)}<C \varepsilon
$$

Since the propagation speed of the system (2.1) is less than or equal to $\sigma$, it follows from (2.4) that

$$
\left\|v_{0}\right\|_{L^{2}\left(B_{R}\right)} \leqq C\|v(\cdot, t)\|_{L^{2}\left(B_{R}+\sigma t\right)}
$$

Write $P(\xi)=\left(p_{i j}(\xi)\right), P(\xi)^{-1}=\left(p^{i j}(\xi)\right), v={ }^{t}\left(v_{1}, \ldots, v_{N}\right)$ and $v_{0}={ }^{t}\left(v_{01}, \ldots\right.$, $\left.v_{0 N}\right)$. By (2.2),

$$
v_{j}(x, t)=\int_{\mathbb{R}^{n}} e^{-i x \cdot \xi} \sum_{k, l=1}^{N} p^{j k}(\xi) e^{-i \lambda_{k}(\xi) t} p_{k l}(\xi) \hat{v}_{0 l}(\xi) d \xi
$$

for $j=1, \ldots, N$. By the assumption (ii), we have

$$
\left|p^{j k}(\xi)\right|,\left|p_{k l}(\xi)\right| \leqq C, 1 \leqq j, k, l \leqq N, \quad \xi \in \mathbb{R}^{n}
$$

Therefore, $p^{j k} p_{k l} \hat{v}_{0 l}$ belongs to $L^{1}\left(\mathbb{R}^{n}\right) \cap L^{2}\left(\mathbb{R}^{n}\right)$. Applying Lemma 3.1 with $A=\left\{y \in \mathbb{R}^{n} ;|y|=\sigma\right\}$ and with $B=B_{R}$, we observe that there exists $T>0$ such that for any $t>T$, the inequality

$$
\|v(\cdot, t)\|_{L^{2}\left(B_{R}+\sigma t \backslash B_{\sigma t}\right)}<\varepsilon
$$

holds. Thus we have

$$
\left\|u_{0}\right\|_{L^{2}\left(\mathbf{R}^{n}\right)} \leqq\left\|v_{0}\right\|_{L^{2}\left(\boldsymbol{B}_{R}\right)}+\left\|u_{0}-v_{0}\right\|_{L^{2}\left(\mathbf{R}^{n}\right)}
$$




$$
\begin{aligned}
& \leqq C\|v(\cdot, t)\|_{L^{2}\left(B_{R}+\sigma t\right)}+\varepsilon \\
& \leqq C\|v(\cdot, t)\|_{L^{2}\left(B_{R}+\sigma t \backslash B_{\sigma t}\right)}+C\|v(\cdot, t)\|_{L^{2}\left(B_{\sigma t}\right)}+\varepsilon
\end{aligned}
$$

by (3.3) and (3.5). Since $u=0$ a.e. in $K$, by (3.4), we get

$$
\|v(\cdot, t)\|_{L^{2}\left(B_{\sigma t}\right)} \leqq\|u(\cdot, t)-v(\cdot, t)\|_{L^{2}\left(\mathbf{R}^{n}\right)} \leqq C \varepsilon
$$

and therefore,

$$
\left\|u_{0}\right\|_{L^{2}\left(\mathbf{R}^{n}\right)}<\left(C^{2}+C+1\right) \varepsilon
$$

by (3.6). This implies that $u_{0}=0$ a.e. in $\mathbf{R}^{n}$, and therefore that $u=0$ a.e. in $\mathbf{R}^{n+1}$. The proof is complete.

Next we turn to the

Proof of Lemma 3.1. Let $\varepsilon>0$ be an arbitrary but fixed number. We choose such a large closed ball $Q$ in $\mathbf{R}^{n}$ that

$$
\left\|f \cdot \chi_{\mathbf{C Q}}\right\|_{L^{2}\left(\mathbf{R}^{n}\right)}<\varepsilon
$$

where $C Q$ is the complement of $Q$ and $\chi_{c} Q$ the characteristic function of the set $C Q$. Since the Lebesgue measure is regular and the measure of $E=\left\{\xi \in \mathbb{R}^{n} \backslash D ;-\nabla \lambda(\xi) \in A\right\}$ equals to zero, there exists an open set $O$ such that

$$
\left\|f \cdot \chi_{o}\right\|_{L^{2}\left(\mathbf{R}^{n}\right)}<\varepsilon \quad \text { and } \quad D \cup E \subset O .
$$

It follows from the continuity of $\nabla \lambda$ that $E$ is closed, and therefore $D \cup E$ is closed. Since $Q \backslash O$ is compact and $(D \cup E) \cap(Q \mid O)=\varnothing, \operatorname{dis}(Q \mid O$, $D \cup E)$, the distance between $Q \backslash O$ and $D \cup E$, is positive.

Let $\rho$ be a function in $C_{0}^{\infty}\left(\mathbf{R}^{n}\right)$ such that $\rho \geqq 0, \int_{\mathbf{R}^{n}} \rho(x) d x=1$ and $\operatorname{supp} \rho \subset B_{1}$. For $\eta>0$, we set $\rho_{\eta}(x)=\eta^{-n} \rho(x / \eta)$ and $f_{\eta}(x)=\rho_{\eta} *\left(f \cdot \chi_{Q \backslash o}\right)(x)$. Let us choose $\eta$ so small that $2 \eta<\operatorname{dis}(D \cup E, Q \backslash O)$ and

$$
\left\|f_{\eta}-f \cdot \chi_{Q \backslash o}\right\|_{L^{2}\left(\mathbf{R}^{n}\right)}<\varepsilon
$$

Since $A$ and $\operatorname{supp} f_{\eta}$ are compact, the set $U=\left\{|\nabla \lambda(\xi)+a| ; \xi \in \operatorname{supp} f_{\eta}\right.$, $a \in A\}$ is compact in $\mathbf{R}^{1}$. Therefore, considering that $\operatorname{supp} f_{\eta} \cap(D \cup E)$ $=\varnothing$, we see that the minimum of $U$ (which will be denoted by $m$ ) is 
positive. This implies that for any $\xi \in \operatorname{supp} f_{\eta}$ and $x \in A$, there exists $j=j(\xi, x)$ such that

$$
\left|\frac{\partial \lambda}{\partial \xi_{j}}(\xi)+x_{j}\right| \geqq \frac{m}{\sqrt{n}}
$$

Let $O^{*}$ be an open bounded set containing $\operatorname{supp} f_{\eta}$ such that $\operatorname{dis}\left(O^{*}\right.$, D) $>0$. By the assumption that $\lambda \in C^{2}\left(\mathbb{R}^{n} \backslash D\right)$,

$$
M=\sup _{\substack{1 \leq j \leq n \\ \xi \in \mathcal{O}^{*}}}\left|\nabla \frac{\partial \lambda}{\partial \xi_{j}}(\xi)\right|<\infty .
$$

For $\xi \in \operatorname{supp} f_{\eta}$, let $B(\xi)$ be the open ball in $\mathbb{R}^{n}$ centered at $\xi$ whose radius equals to $\min \left(\frac{m}{4 \sqrt{n M}}, \frac{1}{2} \operatorname{dis}\left(C O^{*}, \operatorname{supp} f_{\eta}\right)\right)$.

Since $\operatorname{supp} f_{\eta}$ is compact and the family $\left\{B(\xi) ; \xi \in \operatorname{supp} f_{\eta}\right\}$ covers $\operatorname{supp} f_{\eta}$, there exist $\xi^{1}, \xi^{2}, \ldots, \xi^{l}$ such that

$$
\operatorname{supp} f_{\eta} \subset \bigcup_{k=1}^{l} B\left(\xi^{k}\right)
$$

Let $\zeta=\min \left(\frac{m}{4 \sqrt{n} M}, \frac{1}{2} \operatorname{dis}\left(\operatorname{supp} f_{\eta}, \operatorname{CO} *\right)\right), \alpha_{k}=\rho_{\zeta^{*}} * \chi_{B\left(\xi^{k}\right)} \quad$ and $\quad \beta_{k}(\xi)=\alpha_{k}(\xi) /$ $\left(\sum_{j=1}^{l} \alpha_{j}(\xi)\right), \xi \in \bigcup_{j=1}^{l} B\left(\xi^{j}\right), 1 \leqq k \leqq l$.

For $k=1,2, \ldots, l$, let us set

$$
f^{k}(\xi)= \begin{cases}f_{\eta}(\xi) \beta_{k}(\xi) & \xi \in \operatorname{supp} f_{\eta} \\ 0 & \xi \in \complement \operatorname{supp} f_{\eta}\end{cases}
$$

Then, $\sum_{k=1}^{l} f^{k}(\xi)=f_{\eta}(\xi)$ for $\xi \in \mathbb{R}^{n}, f^{k} \in C_{0}^{\infty}\left(\mathbb{R}^{n}\right)$ and for each $k$ and each $x \in A$, there exists $j=j(k, x)$ such that

$$
\left|\frac{\partial \lambda}{\partial \xi_{j}}(\xi)+x_{j}\right| \geqq \frac{m}{2 \sqrt{n}} \quad \text { for } \xi \in \operatorname{supp} f^{k} \text {. }
$$

In fact, for any $y \in \operatorname{supp} f^{k}\left(\subset \operatorname{supp} \alpha_{k}\right)$,

$$
\begin{aligned}
\left|\frac{\partial \lambda}{\partial \xi_{j}}(\xi)-\frac{\partial \lambda}{\partial \xi_{j}}(y)\right| & =\left|\int_{0}^{1} \nabla \frac{\partial \lambda}{\partial \xi_{j}}(s \xi+(1-s) y) \cdot(\xi-y) d s\right| \\
& \leqq M \cdot \frac{m}{2 \sqrt{n} M}=\frac{m}{2 \sqrt{n}} .
\end{aligned}
$$


Therefore, by (3.7),

$$
\begin{aligned}
\left|\frac{\partial \lambda}{\partial \xi_{j}}(y)+x_{j}\right| & \geqq\left|\frac{\partial \lambda}{\partial \xi_{j}}(\xi)+x_{j}\right|-\left|\frac{\partial \lambda}{\partial \xi_{j}}(\xi)-\frac{\partial \lambda}{\partial \xi_{j}}(y)\right| \\
& \geqq \frac{m}{\sqrt{n}}-\frac{m}{2 \sqrt{n}}=\frac{m}{2 \sqrt{n}} \quad \text { for all } y \in \operatorname{supp} f^{k}
\end{aligned}
$$

For $x=a t+b, a \in A, b \in B$, we define

$$
g_{k}(x, t)=\int_{\mathbf{R}^{n}} e^{-i(a \cdot \xi+\lambda(\xi)) t} e^{-i b \cdot \xi} f^{k}(\xi) d \xi
$$

Integrating by parts $d=[n / 2]+1$ times with respect to $\xi_{j}$, we get

$$
g_{k}(x, t)=t^{-d} \int_{\mathbf{R}^{n}} e^{-i(a \cdot \xi+\lambda(\xi)) t} \sum_{q=0}^{d} h_{q}(\xi, b) \frac{\partial^{q} f^{k}}{\partial \xi_{j}^{q}}(\xi) d \xi,
$$

where $h_{q}(\xi, b)$ are represented as linear combinations of products of

$$
\left(a_{j}+\frac{\partial \lambda}{\partial \xi_{j}}(\xi)^{-1}\right),\left(\frac{\partial}{\partial \xi_{j}}\right)^{q^{\prime}} e^{-i b \cdot \xi} \text { and } \frac{\partial^{q^{\prime \prime}+1} \lambda}{\partial \xi_{j}^{q^{\prime \prime}+1}}(\xi), 0 \leqq q^{\prime}, q^{\prime \prime} \leqq d-q
$$

By virtue of (3.8) and the boundedness of $B$,

$$
\left|g_{k}(x, t)\right| \leqq C t^{-d}, \quad k=1,2, \ldots, l
$$

with a constant $C$ independent of $a \in A, b \in B$ and $t$. Therefore,

$$
\left\|\sum_{k=1}^{l} g_{k}(\cdot, t)\right\|_{L^{2}\left(F_{t}\right)} \leqq C\left(c_{1} t+c_{2}\right)^{n / 2} t^{-d}
$$

where $c_{1}=\max (|a| ; a \in A)$ and $c_{2}=\sup (|b| ; b \in B)$. This shows that for sufficiently large $T$,

$$
\left\|\sum_{k=1}^{l} g_{k}(\cdot, t)\right\|_{L^{2}\left(F_{t}\right)}<\varepsilon \quad \text { for } \quad t>T .
$$

Notice that $g(x, t)=\sum_{k=1}^{l} g_{k}(x, t)+\int_{\mathbf{R}^{n}} e^{-i x \cdot \xi-i \lambda(\xi) t}\left(f(\xi)-f_{\eta}(\xi)\right) d \xi . \quad$ By the Parseval's relation, we have

$$
\left\|g(\cdot, t)-\sum_{k=1}^{l} g_{k}(\cdot, t)\right\|_{L^{2}\left(F_{t}\right)}
$$




$$
\begin{aligned}
& \leqq\left(\int_{\mathbb{R}^{n}}\left|\int_{\mathbb{R}^{n}} e^{-i x \cdot \xi-i \lambda(\xi) t}\left(f(\xi)-f_{\eta}(\xi)\right) d \xi\right|^{2} d x\right)^{\frac{1}{2}} \\
& =\left\|f-f_{\eta}\right\|_{L^{2}\left(\mathbb{R}^{n}\right)}<3 \varepsilon .
\end{aligned}
$$

Thus we obtain

$$
\|g(\cdot, t)\|_{L^{2}\left(F_{t}\right)}<4 \varepsilon \quad \text { for } \quad t>T
$$

which completes the proof of Lemma 3.1.

\section{Applications}

We begin with an obvious lemma which is of importance for applications.

Lemma 4.1. Let $\lambda$ be a real-valued function in $C^{2}\left(\mathbb{R}^{n}\right)$ and $H_{\lambda}(\xi)$ be the Hessian $\left(\frac{\partial^{2} \lambda}{\partial \xi_{i} \partial \xi_{j}}(\xi)\right)_{1 \leqq i, j \leqq n}$ of $\lambda$. If $\operatorname{det} H_{\lambda}(\xi) \neq 0$ for all $\xi \in \mathbb{R}^{n}$, then

$$
\operatorname{meas}\left\{\xi \in \mathbb{R}^{n} ;|\nabla \lambda(\xi)|=\sigma\right\}=0 \quad \text { for each } \sigma>0 \text {. }
$$

Proof. It suffices to remark that the Hessian $H_{\lambda}$ is the Jacobian matrix of $\nabla \lambda$ and the set $\left\{\xi \in \mathbf{R}^{n} ;|\nabla \lambda(\xi)|=\sigma\right\}$ is the inverse image of $\nabla \lambda$ of the set $S_{\sigma}=\left\{x \in \mathbb{R}^{n} ;|x|=\sigma\right\}$ of measure zero.

Example 4.2. Consider the Klein-Gordon equation in three space dimensions

$$
\frac{\partial^{2} u}{\partial t^{2}}-\Delta u+\alpha^{2} u=0, \quad \alpha>0
$$

We reduce this equation to the first order symmetric hyperbolic system

$$
\frac{\partial U}{\partial t}=\sum_{j=1}^{3} A_{j} \frac{\partial U}{\partial x_{j}}+L U
$$

where $U={ }^{t}\left(\alpha u, \frac{\partial u}{\partial t}, \frac{\partial u}{\partial x_{1}}, \frac{\partial u}{\partial x_{2}}, \frac{\partial u}{\partial x_{3}}\right), A_{j}$ is the $5 \times 5$ matrix with zeros everywhere except at the $(2, j+2)$ and $(j+2,2)$ elements, whose value is unity, and $L$ is the $5 \times 5$ matrix with only two non-zero elements, 
one at the $(1,2)$ element whose value is $\alpha$ and the other at the $(2,1)$ element whose value is $-\alpha$.

Then, the eigenvalues of $\sum_{j=1}^{3} A_{j} \xi_{j}+i L$ are equal to 0 or $\pm\left(|\xi|^{2}+\alpha^{2}\right)^{\frac{1}{2}}$. Let $H_{\lambda}(\xi)$ denote the Hessian of the function $\lambda(\xi)=\left(|\xi|^{2}+\alpha^{2}\right)^{\frac{1}{2}}$. The determinant of $H_{\lambda}(\xi)$ is in this case

$$
\operatorname{det} H_{\lambda}(\xi)=\alpha^{2}\left(|\xi|^{2}+\alpha^{2}\right)^{-5 / 2}
$$

Hence, $\operatorname{det} H_{\lambda}(\xi) \neq 0$ for all $\xi \in \mathbf{R}^{3}$. In view of Lemma 4.1 , we now easily see that the system (4.1) satisfies the condition (iii).

Since $A_{j}$ and $i L$ are hermitian, the assumption (ii) is valid with some unitary matrix $P$ for the system (4.1).

Now we know that Theorem 2.1 and Corollary 2.2 are applicable to the system (4.1).

Example 4.3. Next we examine the Dirac equation

$$
\frac{\partial u}{\partial t}=\sum_{j=1}^{3} A_{j} \frac{\partial u}{\partial x_{j}}+L u
$$

where

$$
\begin{aligned}
& A_{1}=-\left(\begin{array}{rrrr}
0 & 0 & 0 & 1 \\
0 & 0 & 1 & 0 \\
0 & 1 & 0 & 0 \\
1 & 0 & 0 & 0
\end{array}\right), \quad A_{2}=i\left(\begin{array}{rrrr}
0 & 0 & 0 & 1 \\
0 & 0 & -1 & 0 \\
0 & 1 & 0 & 0 \\
-1 & 0 & 0 & 0
\end{array}\right), \\
& A_{3}=\left(\begin{array}{rrrr}
0 & 0 & -1 & 0 \\
0 & 0 & 0 & 1 \\
-1 & 0 & 0 & 0 \\
0 & 1 & 0 & 0
\end{array}\right) \text { and } L=i \alpha\left(\begin{array}{rrrr}
-1 & 0 & 0 & 0 \\
0 & -1 & 0 & 0 \\
0 & 0 & 1 & 0 \\
0 & 0 & 0 & 1
\end{array}\right), \quad \alpha>0 .
\end{aligned}
$$

An explicit calculation shows that the eigenvalues of $\sum_{j=1}^{3} A_{j} \xi_{j}+i L$ are $\pm\left(|\xi|^{2}+\alpha^{2}\right)^{\frac{1}{2}}$. Therefore, by the same reasoning as above, we conclude that Theorem 2.1 and Corollary 2.2 can be applied to the system (4.2).

Remark. In Lemma 3.1, if we take $A=S_{\delta}$ and replace the assumption meas $\left\{\xi \in \mathbf{R}^{n} \backslash D ;-\nabla \lambda(\xi) \in A\right\}=0$ by $\operatorname{det} H_{\lambda}(\xi) \neq 0$ for all $\xi \in \mathbf{R}^{n}$, then we can prove (3.2) using the asymptotic estimate for the integral 
(3.1) obtained by Littman [4] and by Leblanc [3]. But such a setting is not sufficient to deal with the general case as was seen in the case of Example 4.2.

\section{Necessity of the Condition (iii)}

In this section, we shall show that in one space dimensional case the condition (iii) is necessary in order that the assertion of Theorem 2.1 holds.

We use the same notations as in Theorem 2.1.

Theorem 5.1. Assume $n=1$. If meas $E>0$, then there exist nonzero infinitely differentiable $L^{2}$-solutions of (2.1) which vanish in $K$.

Proof. We shall verify this theorem in case when

$$
\operatorname{meas}\left\{\xi \in \mathbf{R}^{1} \backslash D ; \frac{\partial \lambda_{k}}{\partial \xi}(\xi)=\sigma\right\}>0
$$

for some $k$. The other case is treated in the same way.

We shall write $z=\xi+i \eta, \xi, \eta \in \mathbb{R}^{1}$. The eigenvalues of $A_{1} z+i L$ are branches of analytic functions of $z$ with only algebraic singularities at finite number of points. By the unicity theorem on analytic functions and by (5.1), we see that one of these analytic functions equals to $\sigma z+\mu$ for some $\mu \in \mathbb{R}^{1}$. Here we have used the assumption (ii). Write $\lambda(z)$ $=\sigma z+\mu$. By Kato [2], we know that the eigenprojection $P_{\lambda}(z)$ and eigennilpotent $D_{\lambda}(z)$ corresponding to $\lambda(z)$ are polynomials of $z$ with matrix coefficients since so is $\lambda(z)$. In particular, by the assumption (ii), we have $D_{\lambda}(z)=0$ for all $z \in \mathbb{C}^{1}$.

Let $r>0$ and $f$ be a function in $C_{0}^{\infty}\left(\mathbf{R}^{1}\right)$ with its support in $B_{r}$. By the Paley-Wiener's theorem, the support of the function

$$
u_{0}(x)=\int_{\mathbf{R}^{1}} e^{-i x \xi} P_{\lambda}(\xi) \hat{f}(\xi) d \xi
$$

is contained in $B_{r}$. Hence, the infinitely differentiable solution

$$
u(x, t)=\int_{\mathbf{R}^{1}} e^{-i x \xi} e^{-i(t+r / \sigma)\left(A_{1} \xi+i L\right)} P_{\lambda}(\xi) \hat{f}(\xi) d \xi
$$




$$
\begin{aligned}
& =\int_{\mathbf{R}^{1}} e^{-i x \xi} e^{-i(t+r / \sigma) \lambda(\xi)} P_{\lambda}(\xi) \hat{f}(\xi) d \xi \\
& =e^{-i(t+r / \sigma) \mu} u_{0}(x+\sigma t+r)
\end{aligned}
$$

is supported in $\left\{(x, t) \in \mathbb{R}^{2} ;|x+\sigma t+r| \leqq r\right\}$. Since $P_{\lambda}(\xi) \neq 0$ for all $\xi \in \mathbb{R}^{1}$, we can select $f$ lest $u_{0}$ should vanish identically. The proof is complete.

\section{References}

[1] Goodman, R., One-sided invariant subspaces and domains of uniqueness for hyperbolic equations, Proc. Amer. Math. Soc., 15 (1964), 653-660.

[2] Kato, T., Perturbation theory for linear operators, Springer-Verlag, Berlin, Heidelberg, New York (1966).

[3] Leblanc, N., Un résultat de type Van der Corput, C. R. Acad. Sci. Paris, 267 (1968), 886-887.

[4] Lions, J. L. and Magenes, E., Problèmes aux limites non homogènes et applications, 1, Dunod, Paris (1968).

[5] Littman, W., Fourier transforms of surface-carried measures and differentiability of surface averages, Bull. Amer. Math. Soc., 69 (1963), 766-770.

[6] Mizohata, S., Theory of partial differential equations (in Japanese), Iwanami, Tokyo (1965).

[7] Morawetz, C.S., A uniqueness theorem for the relativistic wave equation, Comm. Pure Appl. Math., 16 (1963), 353-362.

[8] Segal, I. E., Direct formulation of the causality requirements of the $S$-operator, Phys. Rev., 109 (1958), 2191-2198.

[9] Taniguchi, M., A uniqueness theorem for symmetric hyperbolic systems of first order in one space variable, Proc. Japan Acad., 47 (1971), 54-58.

[10] Yosida, K., Functional analysis (second ed.), Springer-Verlag, Berlin, Heidelberg, New York, Kinokuniya, Tokyo (1968). 
